\title{
Papers
}

\section{Histological identification of Helicobacter pylori: comparison of staining methods}

\author{
O Rotimi, A Cairns, S Gray, P Moayyedi, M F Dixon
}

\begin{abstract}
Aim-To determine whether two recently described staining methods (the modified McMullen's and the Helicobacter pylori silver stain HpSS methods) used for the histological identification of $\mathrm{H}$ pylori organisms are superior to two established techniques (the modified Giemsa and anti-H pylori antibody immunostain) in terms of availability, reproducibility, rapidity, sensitivity, and cost.

Methods-Histological sections from 63 paired gastric biopsies from adult patients previously investigated for dyspepsia were stained with the four methods and these were assessed blindly and independently by two observers. Of the 63 patients, 30 were originally negative in all tests for $H$ pylori infection, 30 were positive, and the remaining three cases had discordant results using a combination of five tests (rapid biopsy urease test, urea breath test, culture, serology, and histology).
\end{abstract}

Results-Interobserver agreement was best with the antibody method (98\%), followed by the McMullen's (90\%), Giemsa $(87 \%)$, and $\mathrm{HpSS}(85 \%)$. Of the 60 "gold standard" positive and negative cases, 30 were positive by the modified Giemsa stain, 29 by the McMullen's method, 29 by HpSS, and 30 by the antibody stain. However, there were two false positives with the HpSS method. The modified Giemsa is the cheapest and easiest to perform technically.

Conclusions-When $\boldsymbol{H}$ pylori are present, careful examination will almost always reveal them, whichever of these stains is used. However, the modified Giemsa stain is the method of choice because it is sensitive, cheap, easy to perform, and reproducible.

(F Clin Pathol 2000;53:756-759)

Keywords: Helicobacter organisms; histological identification; staining methods

Infection by Helicobacter pylori has been established as the major cause of chronic gastritis, and is important in the pathogenesis of other gastroduodenal diseases such as peptic ulceration, gastric lymphoma, and gastric cancer. ${ }^{1-3}$ In view of this pathogenetic importance, accurate diagnosis of infection is essential to institute eradication treatment in appropriate cases. Various techniques are used for this purpose, including serology, culture, rapid urease test, ${ }^{13} \mathrm{C}$-urea breath test, and histology.

The histological identification of $H$ pylori infection is now a widely used means of diagnosis. To achieve this, several staining methods are in use. These include modified Giemsa, Warthin-Starry, Gimenez, Genta, and immunohistochemical $H$ pylori antibody stains. Immunohistochemistry is the agreed "gold standard" for histology, being a highly sensitive and specific staining method. ${ }^{4}$ Apart from the Genta staining method, ${ }^{5}$ which is complex and time consuming, the others require an additional routine haematoxylin and eosin stained slide to assess the pathology associated with the infection. A few studies have investigated the sensitivity and specificity of the different staining methods. ${ }^{6-8}$ Although the various methods have their strengths and weaknesses, none has been shown to be superior to others in terms of cost, convenience, and sensitivity.

However, two recently reported methods, a modified McMullen's ${ }^{9}$ and the $H$ pylori silver stain (HpSS), ${ }^{10}$ were each claimed to meet the above standards in comparison with other techniques. The former is a modification of the Gimenez stain described by McMullen et al, ${ }^{11}$ whereas the latter is a silver staining technique, but different from the Warthin-Starry method.

To test the claims of superiority of these methods in the detection of $H$ pylori we compared them with our routine modified Giemsa method and an $H$ pylori immunostain, with emphasis on the factors that influence their general usefulness including availability, reproducibility, rapidity, sensitivity, and cost.

\section{Materials and methods}

Routine formalin fixed and paraffin wax embedded pairs of antral and corpus biopsies from 63 adult patients previously investigated for dyspepsia in whom the $H$ pylori status had been determined by culture, biopsy urease test (CLO), serology, and ${ }^{13} \mathrm{C}$-urea breath test (UBT) in addition to histology were used in our study. Standard histological sections were prepared and stained with modified Giemsa, ${ }^{12}$ the modified McMullen's method, ${ }^{8}$ the new HpSS method, ${ }^{9}$ and an anti-H pylori antibody immunostain (fig 1). Emphasis was placed on 

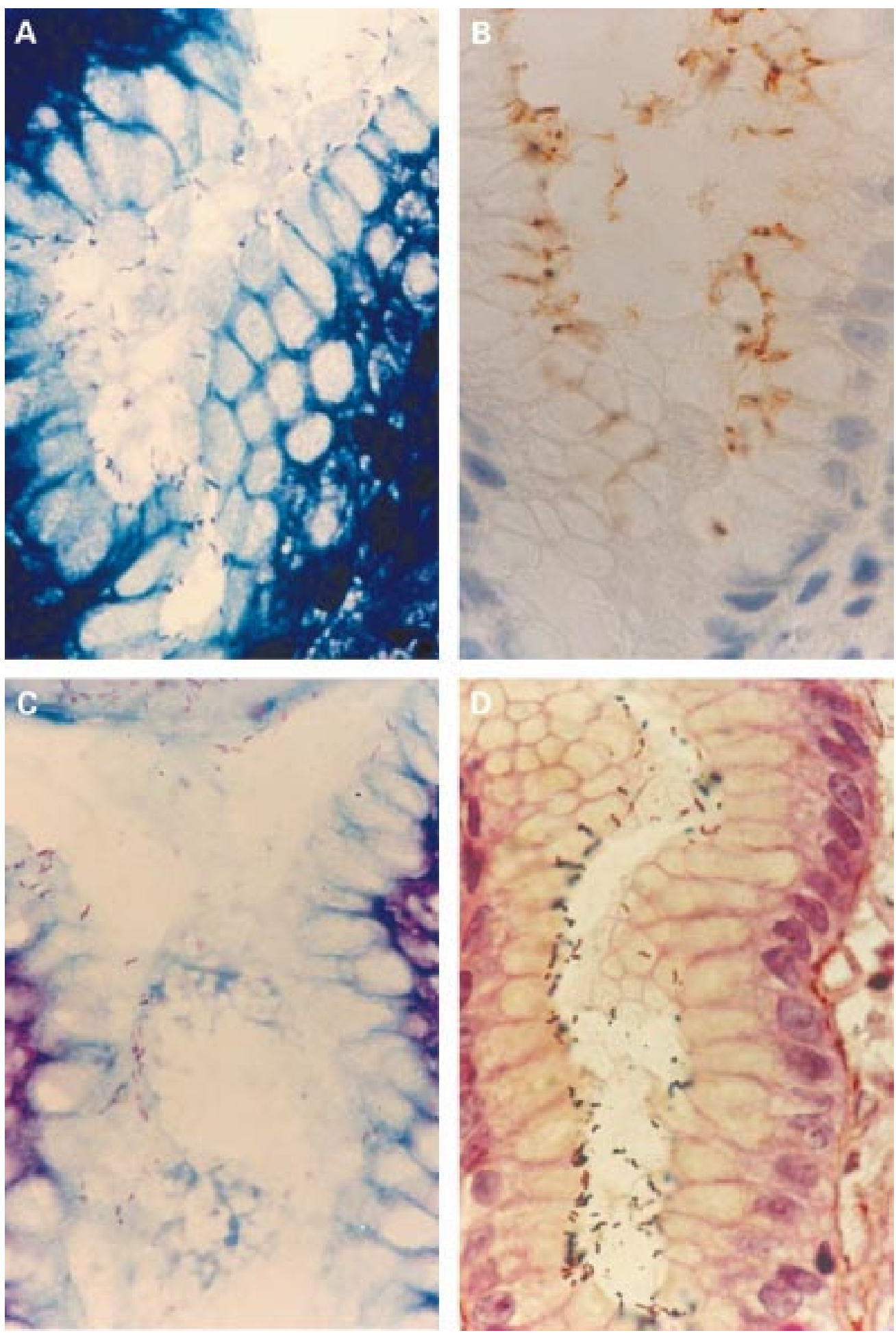

Figure 1 Demonstration of Helicobacter pylori by the four staining methods: (A) modified Giemsa, (B) anti-H pylori antibody immunostain, (C) modified McMullen's method, (D) H pylori silver staining (HpSS) method.

the issues that affect technique-time, availability, and preparation of solutions; staining variations; ease of performance of the techniques; and the reproducibility of the methodologies described.

The avidin-biotin complex (ABC) method was used for the antibody stain using the Duet kit (Dako, Glostrup, Denmark). The sections were pretreated by pressure cooking in $\mathrm{pH} 6.0$ citrate buffer for 90 seconds at full pressure.
The polyclonal $H$ pylori antibody (Dako) was used at a dilution of $1 / 100$.

The stained sections were randomly renumbered before examination by two observers (OR and AC). They were assessed separately without knowledge of previous results, or of the proportions of positive versus negative cases. The results for each observer on each of the staining methods were compared and the interobserver agreement determined. Where 
Table 1 Interobserver agreement and $\kappa$ statistics $(n=60)$

\begin{tabular}{lllll}
\hline Stain & Agreement & $\kappa$ Coefficient & $\begin{array}{l}\text { 95\% confidence } \\
\text { limits }\end{array}$ & Interpretation \\
\hline Modified Giemsa & $0.867(87 \%)$ & 0.733 & 0.49 to 0.98 & Good \\
McMullen's method & $0.900(90 \%)$ & 0.80 & 0.55 to 1.00 & Excellent \\
HpSS & $0.85(85 \%)$ & 0.695 & 0.45 to 0.94 & Good \\
Antibody method & $0.983(98 \%)$ & 0.967 & 0.71 to 1.00 & Excellent, almost perfect \\
\hline
\end{tabular}

HpSS, Helicobacter pylori silver stain.

there was disagreement, the case was reviewed on a double headed microscope to achieve a consensus $H$ pylori status, which was used to compare the methods.

\section{Results}

Of the 63 patients, 30 were originally negative in all tests for $H$ pylori infection, whereas 30 were positive using a combination of five different tests (CLO, UBT, culture, serology, and histology). Infection was considered to be present when three or more tests were positive. The remaining three cases had discordant results, two were initially histology negative but positive by at least one other test (one UBT and serology positive and the other positive by serology only), and the other case was histologically and serologically positive but negative for other tests.

Of the 30 that were previously shown to be infected, 30 were UBT and CLO positive, 27 serology positive, and 29 culture positive. All 30 were previously shown positive by histology using the modified Giemsa stain (a routine procedure with all our gastric biopsies) when examined by several different reporting histopathologists. The patients were aged between 20 and 74 years.

HELICOBACTER PYLORI DETECTION BY THE

STAINING METHODS

The independent observations were tested for chance corrected agreement by $\kappa$ statistics. Table 1 presents the results and their interpretation according to the benchmarks of Svanholm et al. ${ }^{13}$

After consensus examination of the 60 gold standard positive and negative cases, 30 were confirmed positive by the modified Giemsa stain, 29 by the McMullen's method, 29 by the HpSS method, and 30 by the antibody stain. The new silver method, HpSS, gave two false positive results. These could be the result of the stain picking out organisms other than helicobacters.

Of the three cases with previous discordant results, the case only positive by serology was negative for all the staining methods. The other two were negative by the modified Giemsa and McMullen methods; the one previously positive by the UBT test and serology was positive

Table 2 Comparison of the cost and technical times for the methods

\begin{tabular}{llll}
\hline Stain & Relative cost & Average time/slide & $\begin{array}{l}\text { Reproducibility } \\
\text { of technique }\end{array}$ \\
\hline Modified Giemsa & Cheapest & 5 minutes & Good \\
Modified McMullen's & Inexpensive & 10 minutes & Relatively good \\
HpSS & Expensive & 1 hour & Bad \\
Antibody & Expensive & 1 hour & Relatively good
\end{tabular}

HpSS, Helicobacter pylori silver stain. by both the HpSS and antibody methods; the one previously histologically and serologically positive was found positive by the HpSS method only.

COMPARISON OF COSTS AND TECHNICALITIES OF THE METHODS (TABLE 2)

The modified Giemsa stain is very straightforward, inexpensive, and takes about five minutes to perform, excluding the time in solution, and rarely requires repeat stains (none were required in our study). This method is easily reproducible. The major disadvantage is that there is little contrast between organisms and tissue.

The modified McMullen's method is also simple to perform and inexpensive. It takes about 10 minutes of technical time and gives a very good contrast when performed well, making identification of the organism easy. However, because the concentration of the stains and the timings of staining are crucial, there is variation in staining, both within the batch and from batch to batch. Hence, several repeats were required, which increased the time and cost of producing a satisfactory slide for reporting. There was minor difficulty in following the proposed modifications to the original method.

The HpSS method gives a good result because the organisms are coated with the silver stain and therefore look larger, making their identification easy. However, the haematoxylin and eosin counterstaining was rather overpowered by the silver, making the simultaneous assessment of the morphology of the tissue difficult. The technique was quite demanding because glassware had to be thoroughly cleaned to prevent precipitation, and the working solutions took time to prepare. In addition, dropping the AgNOR solution on to the slides was time consuming, and variation occurred within batches and from batch to batch, necessitating several repeats. Overall, it took about one hour to produce a satisfactory slide and it is a fairly expensive technique. The published method did not work initially in our laboratory and we had to seek clarification from the authors before we could achieve satisfactory results.

The antibody stain gives a good result and the method is very reliable. No variations were noted and therefore no repeats were required. The technical time is approximately one hour and the method is fairly expensive, especially because a negative control needs to be done with every slide.

\section{Discussion}

Questions have been asked about the place of histology based on endoscopy, an invasive technique, in the diagnosis of $H$ pylori infection, especially with the availability of noninvasive, more rapid, and less expensive tests, such as serology and the UBT. A recent study has compared the sensitivities and specificities of the UBT, culture, rapid urease test, and histology in the diagnosis of $\mathrm{H}$ pylori infection. ${ }^{14}$ This study reported little difference in their specificities, but histology using the modified 
Giemsa stain with a sensitivity of $98 \%$ was significantly more sensitive than the rest. The added value of histology in providing definitive diagnoses that have important consequences for patient management makes it a justifiable means of diagnosing $H$ pylori infection.

In most cases, $H$ pylori can be recognised in a good haematoxylin and eosin stain. However, the sensitivity of this is low, especially when there are not many bacteria. Given the well documented implications of $H$ pylori infection, correct and prompt diagnosis is essential. Therefore, most laboratories use an additional staining method in the identification of the organism.

We have compared the use of four such stains in the detection of $H$ pylori using a combination of tests to verify infection. There were 30 cases with definite $H$ pylori infection and 30 definite negatives at the time the biopsies were taken. These cases were used to assess interobserver agreement. As can be seen, the observed agreement was best with the antibody method (98\%), then McMullen's (90\%), then Giemsa $(87 \%)$, and lastly HpSS (85\%). The $\kappa$ coefficients followed the same pattern, with the former two emerging as "excellent" and the latter two as "good". Most discrepancies arose through a greater tendency for one observer to make false positive diagnoses. After joint examination, the observers' agreed histological $H$ pylori status differed from the gold standard diagnosis in only two instances. Based on these results it is clear that the antibody method is more reliable than the other methods.

In considering the sensitivities of these techniques it must be remembered that the 30 gold standard positive cases were selected on the basis of multiple tests and all were positive by histology. Thus, it is not surprising that $100 \%$ sensitivity was achieved with the Giemsa stain, even though new sections were examined. The differences in sensitivity between the four methods are minimal and the conclusion to be drawn is that when $H$ pylori are present careful examination will reveal the organisms, no matter which of these stains is used.

From the practical point of view, identification of the organisms is relatively easy with all of the methods, but much easier with the HpSS method because the silver coating makes the organisms larger. This is also true for the McMullen's method because of the staining contrast between the organisms and the adjacent gastric mucosa. The lack of contrast is a disadvantage of the Giemsa technique, but a careful observer should not have problems identifying the organisms. We could not reproduce the purported advantage of the HpSS method of allowing for the simultaneous assessment of the morphology of the biopsies because the silver stain overpowered the haematoxylin and eosin counterstain. This might reflect different technical factors on our part, but it also speaks for the poor reproducibility of the method.

In this era of cost restraint, pathology laboratories must aspire to use the most cost effective method in routine practice. For cost effectiveness, such a method must be relatively cheap, easy to perform, and easy to interpret. No particular method has satisfied these standards entirely. Although we have shown that the most reliable method is the $H$ pylori immunostain, this is offset by the increased expense of reagents and the time taken for each slide. The extra reliability is unlikely to translate into a cost effective clinical benefit. We appreciate that reagents and other costs will vary widely between countries, so our cost comparison has been expressed in relative rather than absolute terms, and we have laid most emphasis on time (labour costs). Such a comparison of the costs/slide of each of these methods shows that the modified Giemsa is the cheapest and the easiest to perform technically. This, coupled with its high sensitivity, ${ }^{14}$ makes it the method of choice.

In conclusion, we have confirmed that the modified Giemsa stain is a reliable, cheap, easy to perform, and convenient histological means of identifying $H$ pylori in gastric biopsies.

1 Dixon MF. Helicobacter pylori and peptic ulceration: histopathological aspects. f Gastroenterol Hepatol 1991;6:125-30.

2 Sipponen P. Helicobacter pylori: a cohort phenomenon. Am f Surg Pathol 1995;19(suppl 1): S30-6.

3 Sipponen P. Gastric cancer-a long-term consequence of Helicobacter pylori infection? Scand $\mathcal{F}$ Gastroenterol 1994; 29(suppl 201):24-7.

4 Ashton-Key M, Diss TC, Isaacson PG. Detection of Helicobacter pylori in gastric biopsy and resection specimens. F Clin Pathol 1996;49:107-11.

5 Genta RM, Robin GO, Graham DY. Simultaneous visualisation of Helicobacter pylori and gastric morphology: a new stain. Hum Pathol 1994;25:221-6.

6 Potters HVPJ, Loffeld RJLF, Stobberingh E, et al. Rapid staining of Campylobacter pyloridis. Histopathology 1987; 11:1223.

7 Madan E, Kemp J, Westblom TU, et al. Evaluation of staining methods for identifying Campylobacter pylori. Am 7 Clin Pathol 1988;90:450-3.

8 Laine L, Lewin DN, Naritoku W, et al. Prospective comparison of H\&E, Giemsa and Genta stains for the diagnosis of Helicobacter pylori. Gastrointest Endosc 1997; 45:463-7.

9 Iwaki H, Sugiyama T, Asaka M. A modified McMullen's staining for Helicobacter pylori: a high contrast, visibly prominent method. Helicobacter 1998;3:45-8.

10 Doglioni C, Turrin M, Macri E, et al. HpSS: a new silver Doglioni C, Turrin M, Macri E, et al. HpSS: a new silver
staining method for Helicobacter pylori. f Clin Pathol 1997;50:461-4.

11 McMullen L, Walker MM, Bain LA, et al. Histological identification of campylobacter using Gimenez technique in gastric antral mucosa. F Clin Pathol 1987;40:464-5.

12 Gray SF, Wyatt JI, Rathbone BJ. Simplified techniques for identifying Campylobacter pyloridis. F Clin Pathol 1986;39: 1279-80.

13 Svanholm H, Starklint H, Gundersen HJG, et al. Reproducibility of histomorphologic diagnoses with special reference to the kappa statistic. APMIS 1989;97:689-98.

14 Moayyedi P, Dixon MF. Any role left for invasive tests? Histology in clinical practice. Gut 1998;43 (suppl 1):S51-5. 